Supporting Information

\title{
Broadband Plasmonic Photocurrent Enhancement from Photosystem I Assembled with Tailored Arrays of Au and Ag Nanodisks
}

Ravi Pamu ${ }^{1,3,6}$, Benjamin J Lawrie ${ }^{4,5}$, Bamin Khomami ${ }^{* 1,2,6}$ and Dibyendu

Mukherjee ${ }^{\star 2,3,6}$

1Department of Mechanical, Aerospace, and Biomedical Engineering, University of

Tennessee, Knoxville, TN, USA. 2Department of Chemical and Biomolecular

Engineering, University of Tennessee, Knoxville, TN, USA. 3Nano-BioMaterials

Laboratory for Energy, Energetics \& Environment (nbml-E3), University of Tennessee,

Knoxville, TN, USA. 4Quantum Heterostructures Group, Materials Science and

Technology Division, Oak Ridge National Laboratory, Oak Ridge, TN, USA 5Quantum

Information Science Group, Physics Division, Oak Ridge National Laboratory, Oak

Ridge, TN, USA 6Sustainable Energy Education and Research Center (SEERC);

University of Tennessee, Knoxville, TN, USA

*E-mail:dmukherj@utk.edu

bkhomami@utk.edu 


\section{Fraction of plasmon and non-plasmon working area calculations:}

\section{Gold Nano-Disks (AuND):}

The height of the AuND determined from the AFM image $\left(\mathrm{H}_{\mathrm{g}}\right)=\sim 41 \mathrm{~nm}$.

The FDTD simulations were performed on nanopatterned designs that were similar to the asfabricated AuND/ITO substrates using EBL. The periodic Nano-Disk patterns indicated pitch $=200 \mathrm{~nm}$, height $=43 \mathrm{~nm}$.

The optimal diameter of the AuND $\left(\mathrm{D}_{\mathrm{g}}\right)=112 \mathrm{~nm}$ where the theoretically predicted absorbance spectra matched that of the measured spectra for the fabricated AuND/ITO patterns.

The circular area of a single Nano-Disk $=9847.04 \mathrm{~nm}^{2}$

The EBL patterning array size $=4 \mathrm{~mm} \times 4 \mathrm{~mm}$

With a $200 \mathrm{~nm}$ pitch, total number of Nano-Disks in the array $(\mathrm{n})=20000 \times 20000=4 \times 10^{8}$

Hence, total available circular area of Nano-Disks $\left(\mathrm{A}_{\mathrm{d}}\right)=9847.04 \mathrm{~nm}^{2} \times 4 \times 10^{8}=3.9388 \mathrm{~mm}^{2}$

The exposed circular area of the working electrode in the electrochemical cell as estimated from based on inner diameter of the working electrode O-ring $\left(\mathrm{A}_{\mathrm{O}}\right)=30.19 \mathrm{~mm}^{2}$

Since PSI is assembled only on the exposed ITO areas without the NDs, the effective working area can be reduced to the exposed ITO working area $\left(A_{g}\right)$.

$\left(\mathrm{A}_{\mathrm{g}}\right)=A_{O}-A_{d}=26.2512 \mathrm{~mm}^{2}$.

The plain ITO area outside the EBL-fabricated ND square array (i.e., the total ITO working area not under LSPR influence) $=30.19 \mathrm{~mm}^{2}-16 \mathrm{~mm}^{2}=14.19 \mathrm{~mm}^{2}$ 


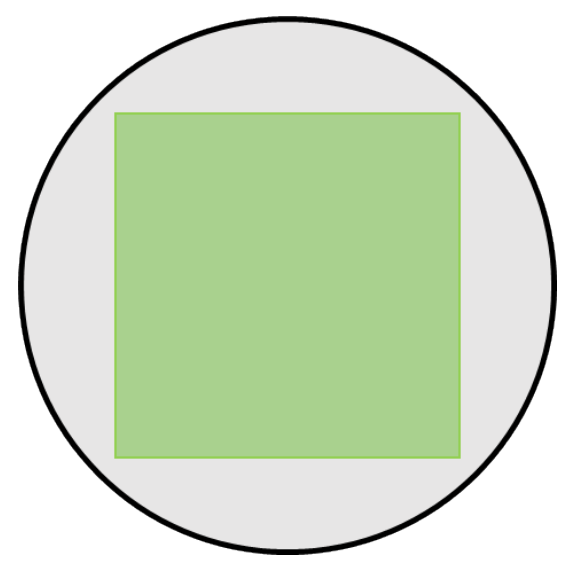

Figure S1. Depicting the electrochemical working area. The square part is nanopattern array of size $4 \mathrm{~mm} \times 4 \mathrm{~mm}$. The gray part is the ITO area $\left(14.19 \mathrm{~mm}^{2}\right)$ located outside the ND array square. The circular part is the O-ring with inner diameter of $6.2 \mathrm{~mm}$.

Fraction of non-plasmonic ITO area $\left(\mathrm{f}_{\mathrm{nP}}\right)=\frac{14.19}{26.2512}=0.54$

Fraction of ITO area under LSPR $\left(f_{P}\right)=\frac{26.2512-14.19}{26.2512}=0.46$

Silver Nano-Disk (AgND):

The analysis method followed here is similar to the method followed above for AuND.

The height of the AgND determined from the AFM image $\left(\mathrm{H}_{\mathrm{s}}\right)=33 \mathrm{~nm}$.

The optimal diameter of the $\operatorname{AgND}\left(\mathrm{D}_{\mathrm{s}}\right)=76 \mathrm{~nm}$

The circular area of a single Nano-Disk $=4534.16 \mathrm{~nm}^{2}$

Total circular area of the Nano-Disks $\left(\mathrm{A}_{\mathrm{s}}\right)=4534.16 \mathrm{~nm}^{2} \times 4 \times 10^{8}=1.813 \mathrm{~mm}^{2}$

Since PSI is assembled only on exposed ITO areas witout the NDs, the working area can be reduced to exposed ITO working area $\left(\mathrm{A}_{\mathrm{s}}\right)$.

$\left(\mathrm{A}_{\mathrm{g}}\right)=A_{O}-A_{d}=28.377 \mathrm{~mm}^{2}$.

The plain ITO area outside the EBL-fabricated ND square array (i.e., the toal ITO working area not under LSPR influence) $=30.19 \mathrm{~mm}^{2}-16 \mathrm{~mm}^{2}=14.19 \mathrm{~mm}^{2}$

Fraction of non-plasmonic ITO area $\left(f_{n P}\right)=\frac{14.19}{28.377}=0.50$

Fraction of ITO area under LSPR $\left(f_{P}\right)=\frac{28.377-14.19}{28.377}=0.50$

2. Plasmon induced photocurrent enhancement factor calculations: 
Photocurrent density of PSI from ITO $_{\text {SAM }}$, i.e., the measured effective photocurrent density of PSI on $\mathrm{ITO}_{\mathrm{SAM}}=\mathbf{P}_{\text {ITO }}$

Photocurrent density of PSI due to the LSPR interactions with AuND or AgND $=\mathbf{P}_{\text {AuND }}$ or $\mathbf{P}_{\text {AgND }}$ (Effective photocurrent density)(AuND/ITO or AgND/ITO) $=\left(f_{P} \times \mathbf{P}_{\text {AuND or AgND }}\right)+\left(f_{\text {nP }} \times \mathbf{P}_{\text {ITo }}\right)$

---------Eqn. (1)

where, (Effective photocurrent density) $(A u N D / I T O$ or $A g N D / I T O)=$ measured effective photocurrent densities of PSI on AuND/ITO or AgND/ITO.

$f_{P}=$ area fraction under direct localized plasmon electric field on AuND/ITO or AgND/ITO

substrate

$f_{n P}=$ non-plasmonic area fraction under indirect localized plasmon electric field on

\section{AuND/ITO or, AgND/ITO substrate}

Eqn (1) is valid since, the measured effective photocurrent density of PSI on AuND/ITO $\mathrm{AgND} / \mathrm{ITO}_{\mathrm{SAM}}$ is emanating from two types of PSI. One set of PSI located inside the nanopatterned array of $4 \mathrm{~mm} \times 4 \mathrm{~mm}$ where the LSPR field is located. Another set of PSI located outside of the LSPR field far away from the nanopatterned array.

The $f_{P}$ and $f_{n P}$ for both the AuND/ITO and AgND/ITO are estimated in the above section 1.

Finally, (Effective Plasmon enhancement factor $)_{\lambda \mathrm{exc}}=\left(\frac{P_{A u N D}}{P_{I T O}}\right)_{\lambda_{\text {exc }}} \operatorname{or}\left(\frac{P_{A g N D}}{P_{I T O}}\right)_{\lambda_{e x c}}$ 
Table S1. The average effective photocurrent values and enhancement factors for each substrate with respect to the excitation wavelength.

\begin{tabular}{|c|c|c|c|c|c|c|c|c|}
\hline $\mathrm{nm}$ & $\begin{array}{l}\text { ITO }_{\text {SAM }} \\
\left(\mathrm{nA} / \mathrm{cm}^{2}\right. \\
)\end{array}$ & $\begin{array}{c}\text { AuND/ITO } \\
\mathrm{MA} \\
\left(\mathrm{nA} / \mathrm{cm}^{2}\right)\end{array}$ & $\begin{array}{c}\text { AgND/ITO }_{S A} \\
M \\
\left(\mathrm{nA} / \mathrm{cm}^{2}\right)\end{array}$ & Р Іто & $P_{\text {AuND }}$ & $P_{\text {AgND }}$ & $\frac{P_{\text {AuND }}}{P_{\text {ITO }}}$ & $\frac{P_{\text {AgND }}}{P_{\text {ITO }}}$ \\
\hline $\begin{array}{l}81 \\
0\end{array}$ & 10.5 & 20.5 & 16.7 & $\begin{array}{l}10 . \\
5\end{array}$ & 32.36 & 22.91 & 3.10 & 2.19 \\
\hline $\begin{array}{l}73 \\
0 \\
\end{array}$ & 16.0 & 28.6 & 29.2 & $\begin{array}{l}16 . \\
0\end{array}$ & 43.40 & 42.37 & 2.71 & 2.64 \\
\hline $\begin{array}{l}68 \\
0\end{array}$ & 23.5 & 86.0 & 51.5 & $\begin{array}{l}23 . \\
5\end{array}$ & $\begin{array}{l}159.1 \\
7\end{array}$ & 79.43 & 6.77 & 3.38 \\
\hline $\begin{array}{l}66 \\
0 \\
\end{array}$ & 20.0 & 67.5 & 72.0 & $\begin{array}{l}20 . \\
0\end{array}$ & $\begin{array}{l}123.3 \\
0\end{array}$ & 124.0 & 6.16 & 6.20 \\
\hline $\begin{array}{l}56 \\
5\end{array}$ & 13.0 & 40.0 & 120.0 & $\begin{array}{l}13 . \\
0\end{array}$ & 71.63 & 227.0 & 5.51 & 17.46 \\
\hline $\begin{array}{l}47 \\
0 \\
\end{array}$ & 16.6 & 27.1 & 153.0 & $\begin{array}{l}16 . \\
6 \\
\end{array}$ & 39.40 & $\begin{array}{l}289.5 \\
0\end{array}$ & 2.36 & 17.37 \\
\hline $\begin{array}{l}45 \\
5 \\
\end{array}$ & 29.6 & 62.7 & 192.0 & $\begin{array}{l}29 . \\
6 \\
\end{array}$ & $\begin{array}{l}101.5 \\
0\end{array}$ & $\begin{array}{l}354.6 \\
0 \\
\end{array}$ & 3.42 & 11.95 \\
\hline $\begin{array}{l}42 \\
0 \\
\end{array}$ & 24.5 & 54.0 & 110.6 & $\begin{array}{l}24 . \\
5 \\
\end{array}$ & 88.58 & $\begin{array}{l}196.8 \\
0\end{array}$ & 3.61 & 8.03 \\
\hline $\begin{array}{l}39 \\
5\end{array}$ & 23.0 & 50.0 & 28.0 & $\begin{array}{l}23 . \\
0\end{array}$ & 81.88 & 33.12 & 3.56 & 1.44 \\
\hline
\end{tabular}

\section{FDTD simulated absorbance spectrum:}



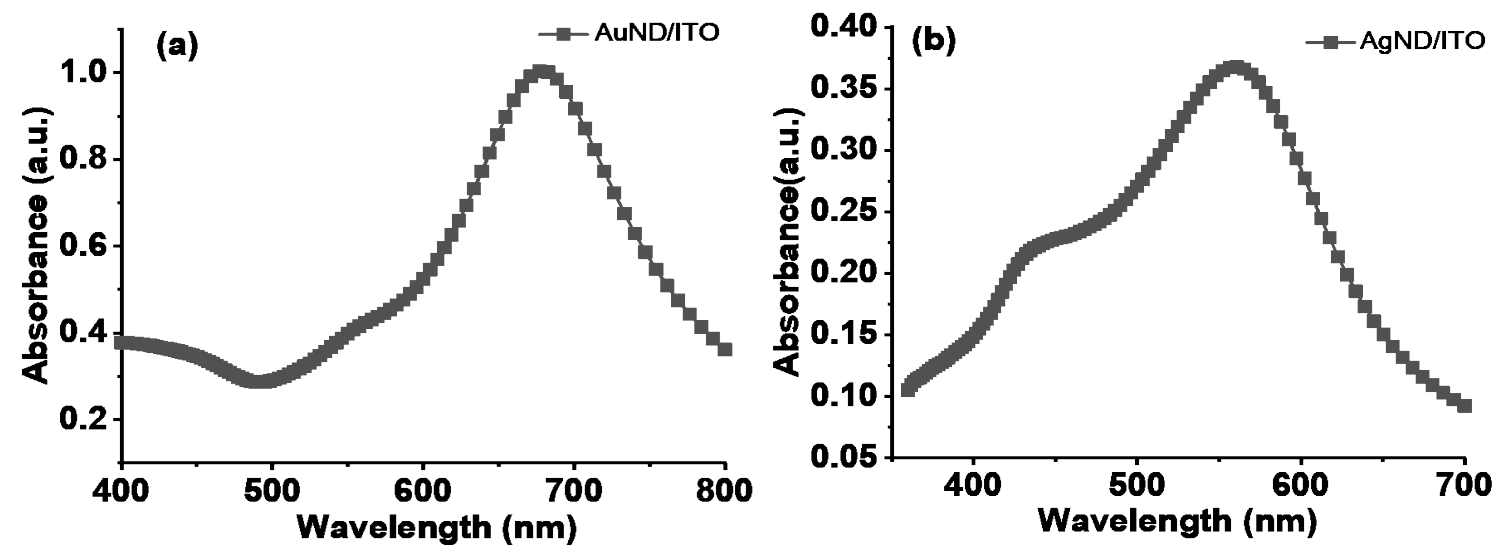

Figure S2. (a) FDTD simulated LSPR absorbance spectrm for AuND/ITO in water (b) FDTD simulated LSPR absorbance spectrum for AgND/ITO in water. The background refractive index of 1.33 is used to reflect the water sorrounding. The AuND/ITO and AgND/ITO absorbance maximum are located at $677 \mathrm{~nm}$ and $561 \mathrm{~nm}$ respectively.
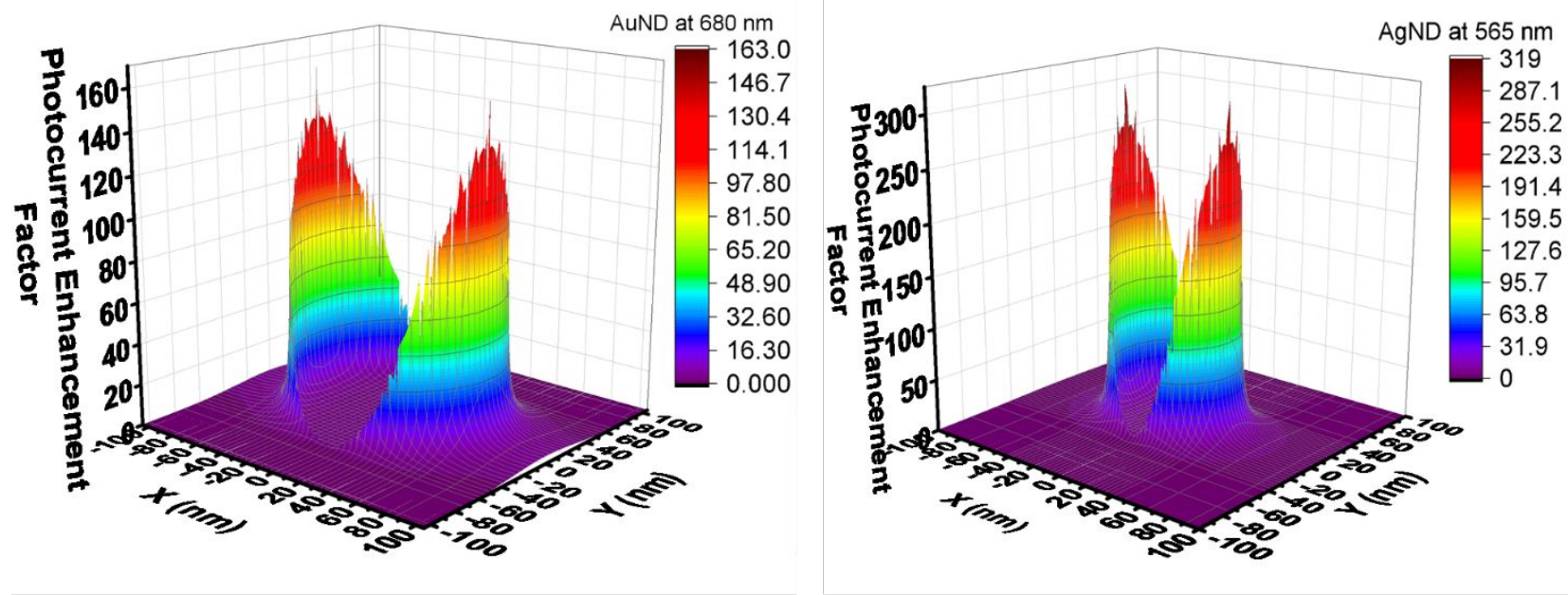

Figure S3. The spatial distribution of experimental enhancement factors for AuND/ITO $\mathrm{SAM}_{\text {and }}$ AgND/ITO ${ }_{\text {SAM }}$ (at $680 \mathrm{~nm}$ and $565 \mathrm{~nm}$ excitations respectively), based on both the experimental Photocurrent density measurements and FDTD simulated LSPR field enhancement distributions 

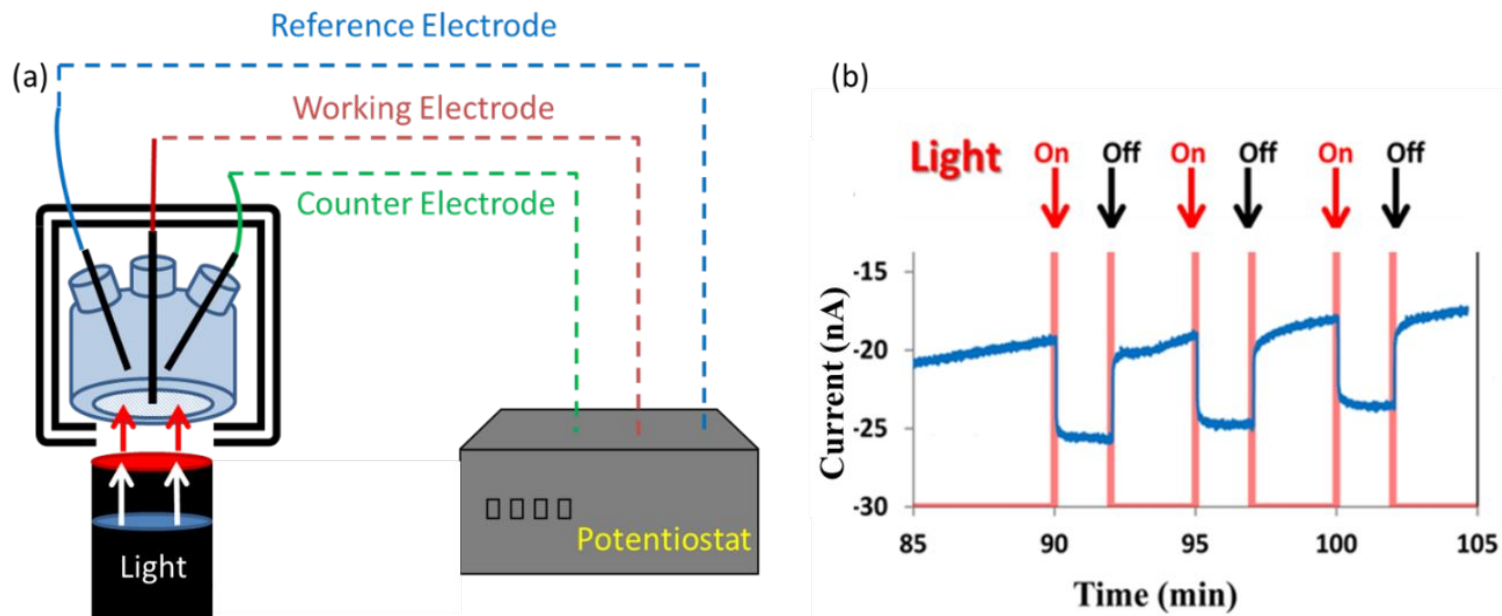

Figure S4. Representative experimental setup for photocurrent measurements. (a) standard 3electrode electrochemical cell connected to potentiostat. (b) representative photocurrent measurments during light on-off cycles. 